\title{
Excess Volume and Deviation in Viscosities of Binary Mixtures of $o$-Dichlorobenzene and o-Chlorophenol with Ethyl Methyl Ketone, Methyl Isobutyl Ketone, Propiophenone and Cyclohexane
}

\author{
A. B. SAWANT ${ }^{\mathrm{a}^{*}}$, MEHDI HASAN ${ }^{\mathrm{a}}$ and V J. NAUKUDKAR ${ }^{\mathrm{b}}$ \\ ${ }^{\mathrm{a}}$ Department of Chemistry, M S G College, Affiliated to University of Pune, Malegaon \\ Camp 423 105, Maharashtra, India \\ ${ }^{\mathrm{b}}$ Department of Chemistry, K. V. N. Naik, Arts, Commerce \& Science College Nashik, \\ Nashik 422002 Maharashtram, India \\ absawant@rediffmail.com
}

Received 27 July 2012 / Accepted 4 September 2012

\begin{abstract}
The densities and viscosities of binary mixtures of $o$-dichlorobenzene and $o$-chlorophenol with ethyl methyl ketone, methyl isobutyl ketone, propiophenone and cyclohexane have been measured at room temperature. The data have been utilized to compute the excess volumes and deviation in viscosities. The results have been interpreted in terms of molecular interactions existing between the components of the mixtures.
\end{abstract}

Keywords: Excess molar volume, Viscosity deviation, o-Dichlorobenzene, o-Chlorophenol, Ketones, Cyclohexane, Molecular interactions

\section{Introduction}

The composition dependence of thermodynamic properties has proved to be very useful tools in understanding the nature and extent of pattern of molecular aggregation resulting from intermolecular interaction between components ${ }^{1,2}$. In the present paper binary liquid mixture of $o$-dichlorobenzene (ODB) and $o$-chlorophenol (OCP) with ethyl methyl ketone (EMK), methyl isobutyl ketone (MIBK), propiophenone (PN) and cyclohexane (CYN) have been studied.

The thermodynamic and transport properties of $o$-dichlorobenzene (ODB) and $o$-chlorophenol (OCP) with above ketones have been studied over the entire composition range at $298.15 \mathrm{~K}$

\section{Experimental}

The entire chemical used was of A. R. grade with purity $>99 \%$ are used as such. The purity of liquid was checked by comparing experimental values of densities and viscosities of these 
liquids with those reports in the literature (Table 1$)^{3-9}$. Binary mixtures were prepared by mixing a known mass of each liquid in an airtight, stoppered glass bottle. The masses were recorded on digital balance (SHIMANZU AUX 220) to an accuracy of $\pm 1 \times 10^{-4} \mathrm{~g}$. The estimated error in mole fraction was $<1 \times 10^{-4}$. Care was taken to avoid contamination during mixing.

Table 1. Comparison of experimental data of $\rho$ and $\eta$ with literature at $298.15 \mathrm{~K}$

\begin{tabular}{ccccc}
\hline \multirow{2}{*}{ Liquids } & \multicolumn{2}{c}{$\rho \times 10^{-3} \mathrm{~kg} . \mathrm{m}^{-3}$} & \multicolumn{2}{c}{$\eta \times 10^{3} \mathrm{~kg} . \mathrm{m}^{-1} \cdot \mathrm{s}^{-1}$} \\
\cline { 2 - 5 } & Exp & Lit & Exp & Lit \\
\hline ODB & 1.2995 & $1.3003^{3}$ & 1.326 & $1.324^{3}$ \\
OCP & 1.2577 & $1.2572^{3}$ & 3.479 & -- \\
EMK & 0.7991 & $0.7999^{5}$ & 0.405 & $0.4053^{6}$ \\
PN & 1.0082 & $1.0087^{3}$ & 1.700 & -- \\
MIBK & 0.7963 & $0.7963^{4}$ & 0.542 & $0.542^{7}$ \\
CYN & 0.7737 & $0.7738^{8}$ & 0.881 & $0.886^{9}$ \\
\hline
\end{tabular}

In this present study a specific gravity bottle ${ }^{10} /$ single stem pycnometer ${ }^{11}$ was used for the density measurements. The specific gravity bottle was calibrated with freshly prepared triply distilled water. The density measurements for each experimental liquid were repeated three to four times. This procedure enabled us to get an uncertainty of $\pm 5 \times 10^{-4} \mathrm{gm} / \mathrm{cm}^{3}$ in density measurements.

The density measurements for each experimental liquid were repeated at least three to four times and the results averaged. This procedure enabled us to get an uncertainty of \pm 0.0005 g. $\mathrm{cm}^{-3}$ in density measurements.

\section{Theory}

The excess molar volume $\left(\mathrm{V}^{\mathrm{E}}\right)$ can be computed from experimental density data using the relationship.

$$
\mathrm{V}^{\mathrm{E}}=\mathrm{x}_{1} \mathrm{M}_{1}\left[1 / \rho_{\mathrm{m}}-1 / \rho_{1}\right]+\mathrm{x}_{2} \mathrm{M}_{2}\left[1 / \rho_{\mathrm{m}}-1 / \rho_{2}\right]
$$

Where $\mathrm{x}_{\mathrm{i}}, \mathrm{M}_{\mathrm{i}}$ and $\rho_{\mathrm{i}}$ designate the mole fraction, the molecular weight and the density of the component i. $\rho_{\mathrm{m}}$ is the density of mixture. The Ostwald viscometer was calibrated with triply distilled water at $298.15 \mathrm{~K}$. A viscometer was selected having a flow time of approximately 100-300 seconds at $298.15 \mathrm{~K}$. The flow time measurement was repeated a number of times (usually 5-6 times). The difference reading did not deviate from the mean by more than $0.2 \mathrm{~s}$. To determine the influence of temperature on viscosity, the time of out flow was measured at $298.15 \mathrm{~K}$. From the densities and times of flow, absolute viscosities of the liquid mixtures were calculated. Deviations in viscosities $(\Delta \eta)$ were obtained as follows:

$$
\Delta \eta=\eta_{\text {mix }}-\left(x_{1} \eta_{1}+x_{2} \eta_{2}\right)
$$

Where $\eta_{1}$ and $\eta_{2}$ are the viscosities of pure components 1 and 2 , respectively and $\eta_{\text {mix }}$ is the viscosity of the liquid mixture.

\section{Results and Discussion}

\section{$V^{E}$ and $\Delta \eta$ studies}

The derived parameter data $\mathrm{V}^{\mathrm{E}}$ and $\Delta \eta$ at the experimental temperamental are listed in Table 2 and 3.The density and viscosities for the binary mixtures of ODB with EMK, PN, MIBK and CYN are listed in Table 2 and OCP with EMK, PN, MIBK and CYN are listed in Table 3 at atmospheric pressure and at $298.15 \mathrm{~K}$. Figures 1 to 4 exhibit the variation of $\mathrm{V}^{\mathrm{E}} / \Delta \eta$ of ODB/OCP with ketones at $298.15 \mathrm{~K}$ and atmospheric pressure. 
Table 2. $\rho, \eta, \mathrm{V}^{\mathrm{E}}$ and $\Delta \eta$ of binary liquid mixture at $298.15 \mathrm{~K}$

\begin{tabular}{|c|c|c|c|c|}
\hline $\mathrm{x}_{1}$ & $\begin{array}{l}\rho \times 10^{-3} \\
\mathrm{~kg} \cdot \mathrm{m}^{-3}\end{array}$ & $\begin{array}{c}\eta \times 10^{3} \\
\mathrm{~kg} \cdot \mathrm{m}^{-1} \cdot \mathrm{s}^{-1}\end{array}$ & $\begin{array}{l}\mathrm{V}^{\mathrm{E}} \times 10^{6} \\
\mathrm{~m}^{3} \cdot \mathrm{mol}^{-1}\end{array}$ & $\begin{array}{l}\Delta \eta \times 10^{3} \\
\mathrm{~kg} \cdot \mathrm{m}^{-1} \cdot \mathrm{s}^{-1}\end{array}$ \\
\hline \multicolumn{5}{|c|}{$\mathrm{ODB}\left(\mathrm{x}_{1}\right)+\mathrm{EMK}\left(1-\mathrm{x}_{1}\right)$} \\
\hline 0.0820 & 0.8570 & 0.456 & -1.655 & -0.024 \\
\hline 0.1674 & 0.9200 & 0.517 & -2.843 & -0.036 \\
\hline 0.2564 & 0.9782 & 0.579 & -3.477 & -0.049 \\
\hline 0.3491 & 1.0347 & 0.647 & -3.918 & -0.061 \\
\hline 0.4458 & 1.0906 & 0.723 & -4.286 & -0.067 \\
\hline 0.5468 & 1.1459 & 0.811 & -4.588 & -0.066 \\
\hline 0.6524 & 1.1946 & 0.909 & -4.330 & -0.057 \\
\hline 0.7629 & 1.2362 & 1.016 & -3.495 & -0.044 \\
\hline 0.8786 & 1.2776 & 1.131 & -2.656 & -0.028 \\
\hline \multicolumn{5}{|c|}{$\operatorname{ODB}\left(\mathrm{x}_{1}\right)+\mathrm{PN}\left(1-\mathrm{x}_{1}\right)$} \\
\hline 0.1157 & 1.0410 & 1.662 & -0.711 & 0.013 \\
\hline 0.2274 & 1.0793 & 1.630 & -1.775 & 0.029 \\
\hline 0.3353 & 1.1158 & 1.598 & -2.523 & 0.045 \\
\hline 0.4397 & 1.1465 & 1.564 & -2.586 & 0.056 \\
\hline 0.5407 & 1.1745 & 1.525 & -2.368 & 0.062 \\
\hline 0.6384 & 1.1995 & 1.481 & -1.864 & 0.060 \\
\hline 0.7331 & 1.2253 & 1.432 & -1.468 & 0.053 \\
\hline 0.8248 & 1.2503 & 1.380 & -1.033 & 0.041 \\
\hline 0.9137 & 1.2751 & 1.327 & -0.604 & 0.027 \\
\hline \multicolumn{5}{|c|}{$\operatorname{ODB}\left(\mathrm{x}_{1}\right)+\operatorname{MIBK}\left(1-\mathrm{x}_{1}\right)$} \\
\hline 0.1097 & 0.8591 & 0.629 & -1.697 & -0.010 \\
\hline 0.2170 & 0.9210 & 0.696 & -3.151 & -0.018 \\
\hline 0.3221 & 0.9809 & 0.767 & -4.155 & -0.021 \\
\hline 0.4250 & 1.0380 & 0.840 & -4.706 & -0.020 \\
\hline 0.5258 & 1.0926 & 0.914 & -4.927 & -0.016 \\
\hline 0.6245 & 1.1457 & 0.989 & -4.965 & -0.010 \\
\hline 0.7212 & 1.1937 & 1.073 & -4.525 & 0.006 \\
\hline 0.8160 & 1.2354 & 1.147 & -3.551 & 0.014 \\
\hline 0.9089 & 1.2702 & 1.215 & -2.041 & 0.016 \\
\hline \multicolumn{5}{|c|}{$\operatorname{ODB}\left(\mathrm{x}_{1}\right)+\mathrm{CYN}\left(1-\mathrm{x}_{1}\right)$} \\
\hline 0.0967 & 0.8349 & 0.888 & -1.500 & -0.013 \\
\hline 0.1942 & 0.9080 & 0.916 & -3.858 & -0.023 \\
\hline 0.2923 & 0.9779 & 0.946 & -5.546 & -0.033 \\
\hline 0.3912 & 1.0467 & 0.979 & -6.905 & -0.040 \\
\hline 0.4908 & 1.1128 & 1.019 & -7.865 & -0.039 \\
\hline 0.5911 & 1.1727 & 1.064 & -8.176 & -0.035 \\
\hline 0.6922 & 1.2185 & 1.111 & -7.263 & -0.028 \\
\hline 0.7940 & 1.2553 & 1.159 & -5.653 & -0.020 \\
\hline 0.8966 & 1.2742 & 1.214 & -2.603 & -0.008 \\
\hline
\end{tabular}


Table 3. $\rho, \eta, \mathrm{V}^{\mathrm{E}}$ and $\Delta \eta$ of binary liquid mixtures at $298.15 \mathrm{~K}$

\begin{tabular}{|c|c|c|c|c|}
\hline $\mathrm{x}_{1}$ & $\begin{array}{l}\rho \times 10^{-3} \\
\mathrm{~kg} \cdot \mathrm{m}^{-3}\end{array}$ & $\begin{array}{c}\eta \times 10^{3} \\
\mathrm{~kg} \cdot \mathrm{m}^{-1} \cdot \mathrm{s}^{-1}\end{array}$ & $\begin{array}{l}\mathrm{V}^{\mathrm{E}} \times 10^{6} \\
\mathrm{~m}^{3} \cdot \mathrm{mol}^{-1}\end{array}$ & $\begin{array}{l}\Delta \eta \times 10^{3} \\
\mathrm{~kg} \cdot \mathrm{m}^{-1} \cdot \mathrm{s}^{-1}\end{array}$ \\
\hline \multicolumn{5}{|c|}{$\mathrm{OCP}\left(\mathrm{x}_{1}\right)+\mathrm{EMK}\left(1-\mathrm{x}_{1}\right)$} \\
\hline 0.0897 & 0.0897 & 0.0897 & 0.0897 & 0.0897 \\
\hline 0.1816 & 0.1816 & 0.1816 & 0.1816 & 0.1816 \\
\hline 0.2755 & 0.2755 & 0.2755 & 0.2755 & 0.2755 \\
\hline 0.3717 & 0.3717 & 0.3717 & 0.3717 & 0.3717 \\
\hline 0.4701 & 0.4701 & 0.4701 & 0.4701 & 0.4701 \\
\hline 0.5710 & 0.5710 & 0.5710 & 0.5710 & 0.5710 \\
\hline 0.6743 & 0.6743 & 0.6743 & 0.6743 & 0.6743 \\
\hline 0.7802 & 0.7802 & 0.7802 & 0.7802 & 0.7802 \\
\hline 0.8887 & 0.8887 & 0.8887 & 0.8887 & 0.8887 \\
\hline \multicolumn{5}{|c|}{$\mathrm{OCP}\left(\mathrm{x}_{1}\right)+\mathrm{PN}\left(1-\mathrm{x}_{1}\right)$} \\
\hline 0.1261 & 0.1261 & 0.1261 & 0.1261 & 0.1261 \\
\hline 0.2451 & 0.2451 & 0.2451 & 0.2451 & 0.2451 \\
\hline 0.3575 & 0.3575 & 0.3575 & 0.3575 & 0.3575 \\
\hline 0.4640 & 0.4640 & 0.4640 & 0.4640 & 0.4640 \\
\hline 0.5649 & 0.5649 & 0.5649 & 0.5649 & 0.5649 \\
\hline 0.6607 & 0.6607 & 0.6607 & 0.6607 & 0.6607 \\
\hline 0.7518 & 0.7518 & 0.7518 & 0.7518 & 0.7518 \\
\hline 0.8385 & 0.8385 & 0.8385 & 0.8385 & 0.8385 \\
\hline 0.9212 & 0.9212 & 0.9212 & 0.9212 & 0.9212 \\
\hline \multicolumn{5}{|c|}{$\operatorname{OCP}\left(\mathrm{x}_{1}\right)+\operatorname{MIBK}\left(1-\mathrm{x}_{1}\right)$} \\
\hline 0.1196 & 0.1196 & 0.1196 & 0.1196 & 0.1196 \\
\hline 0.2342 & 0.2342 & 0.2342 & 0.2342 & 0.2342 \\
\hline 0.3439 & 0.3439 & 0.3439 & 0.3439 & 0.3439 \\
\hline 0.4491 & 0.4491 & 0.4491 & 0.4491 & 0.4491 \\
\hline 0.5502 & 0.5502 & 0.5502 & 0.5502 & 0.5502 \\
\hline 0.6472 & 0.6472 & 0.6472 & 0.6472 & 0.6472 \\
\hline 0.7405 & 0.7405 & 0.7405 & 0.7405 & 0.7405 \\
\hline 0.8303 & 0.8303 & 0.8303 & 0.8303 & 0.8303 \\
\hline 0.9167 & 0.9167 & 0.9167 & 0.9167 & 0.9167 \\
\hline \multicolumn{5}{|c|}{$\operatorname{OCP}\left(\mathrm{x}_{1}\right)+\operatorname{CYN}\left(1-\mathrm{x}_{1}\right)$} \\
\hline 0.1056 & 0.1056 & 0.1056 & 0.1056 & 0.1056 \\
\hline 0.2100 & 0.2100 & 0.2100 & 0.2100 & 0.2100 \\
\hline 0.3130 & 0.3130 & 0.3130 & 0.3130 & 0.3130 \\
\hline 0.4148 & 0.4148 & 0.4148 & 0.4148 & 0.4148 \\
\hline 0.5153 & 0.5153 & 0.5153 & 0.5153 & 0.5153 \\
\hline 0.6146 & 0.6146 & 0.6146 & 0.6146 & 0.6146 \\
\hline 0.7127 & 0.7127 & 0.7127 & 0.7127 & 0.7127 \\
\hline 0.8096 & 0.8096 & 0.8096 & 0.8096 & 0.8096 \\
\hline 0.9054 & 0.9054 & 0.9054 & 0.9054 & 0.9054 \\
\hline
\end{tabular}

\section{$\mathrm{ODB}+$ ketones}

From Figure 1, $\mathrm{V}^{\mathrm{E}}$ are negative for binaries ODB + EMK, ODB + PN, ODB + MIBK, ODB $+\mathrm{CYN}$ at $298.15 \mathrm{~K}$ and atmospheric pressure over the entire range of composition. ODB + CYN for which the $\mathrm{V}^{\mathrm{E}}$ values are more negative, which may be due to the formation of weak molecular complexes, when compared to all remaining mixtures. 
Figure 2 shows the variation in $\Delta \eta$ for binaries ODB + EMK, ODB + PN, ODB + MIBK at $298.15 \mathrm{~K}$. ODB + EMK and ODB + CYN show negative $\Delta \eta$. ODB + MIBK show negative as well positive deviation. $\Delta \eta$ values shows negative may be due to unequal molecular size of the constituent molecules of the mixture where dispersion forces are dominant ${ }^{12}$. ODB + PN show positive deviation. The positive values of $\Delta \eta$ in these mixtures can be interpreted in terms of strong interactions between unlike molecules i.e. in ODB + PN system.

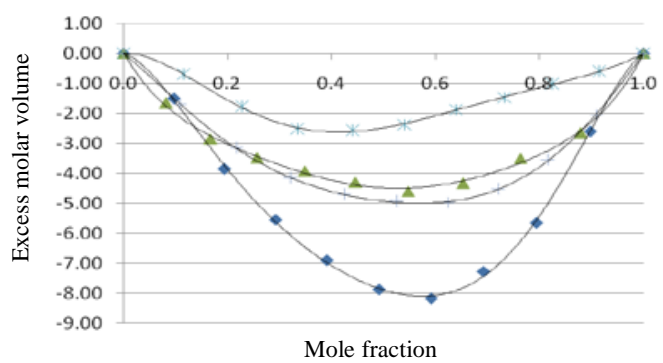

Figure 1. Variation of $\mathrm{V}^{\mathrm{E}}$ for $\left(\mathrm{x}_{1}\right)$ ODB + (1-x) PN (Ж), EMK ( $\triangle$ ), MIBK (x) and CYN (匹) at $298.15 \mathrm{~K}$

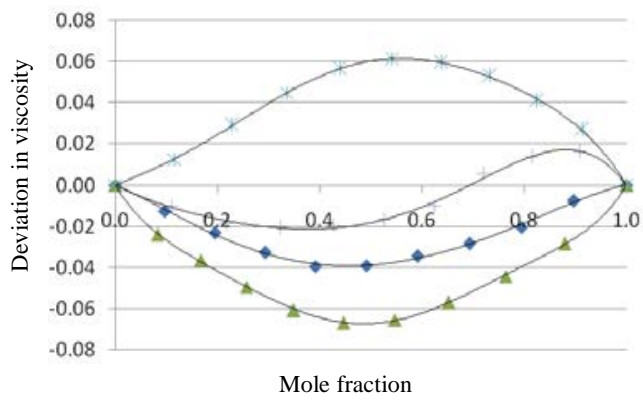

Figure 2. Variation of $\Delta \eta$ for $\left(\mathrm{x}_{1}\right)$ ODB $+(1-\mathrm{x}) \operatorname{PN}(Ж)$, MIBK (+), EMK ( $\triangle$ ) and CYN (匹) at $298.15 \mathrm{~K}$

OCP + ketones

Figures 3 shows the variation in $\mathrm{V}^{\mathrm{E}}$ for binaries OCP + EMK, OCP + MIBK, OCP + PN, OCP + CYN at 298.15 K. OCP + PN show less negative deviation and OCP + CYN shows more negative $\mathrm{V}^{\mathrm{E}}$.

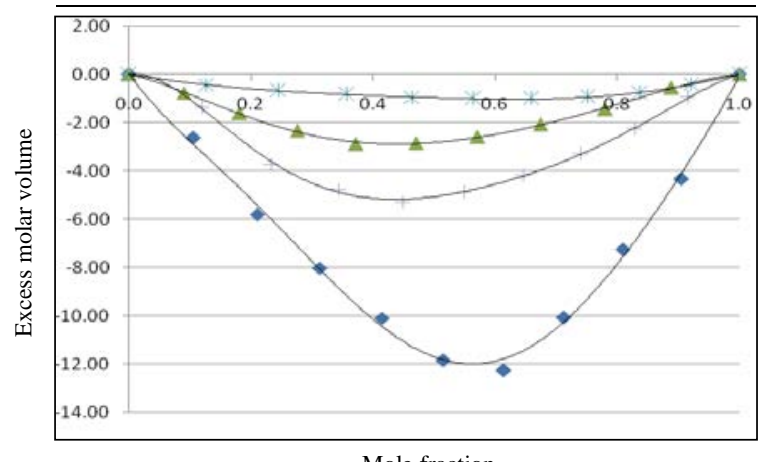

Mole fraction

Figure 3. Variation of $\mathrm{V}^{\mathrm{E}}$ for $\left(\mathrm{x}_{1}\right)$ OCP + (1-x) PN (Ж), EMK ( $\triangle$ ), MIBK (x) and CYN (匹) at $298.15 \mathrm{~K}$ 
The $\mathrm{V}^{\mathrm{E}}\left(\mathrm{x}_{1} \approx 0.5\right)$ shows the order: $\mathrm{OCP}+\mathrm{PN}>\mathrm{OCP}+\mathrm{EMK}>\mathrm{OCP}+\mathrm{MIBK}>\mathrm{OCP}+$ $\mathrm{CYN}$. Negative $\mathrm{V}^{\mathrm{E}}$ reveals the existence of specific interaction between unlike molecules ${ }^{13}$. The interaction takes place between a lone pair of electrons of the oxygen atom of ketone and $\mathrm{Cl}, \mathrm{O}-\mathrm{H}$ group ${ }^{14}$. This may also be due to the formation of weak molecular complexes due change of free volumes in the real mixture because of interstitial accommodation of ketone molecules into cluster of OCP molecules.

Figure 4 gives the variation of $\Delta \eta$ for binaries OCP + EMK, OCP + PN, OCP + MIBK, OCP + CYN at $298.15 \mathrm{~K}$ over the entire composition range. OCP + CYN show negative deviation. OCP + MIBK and OCP + EMK both show negative as well positive deviations. Negative $\Delta \eta$ may be ascribed due to dominating dispersion forces. OCP + PN show positive deviation. The positive values of $\Delta \eta$ in these mixtures can be interpreted in terms of strong interactions between unlike molecules.

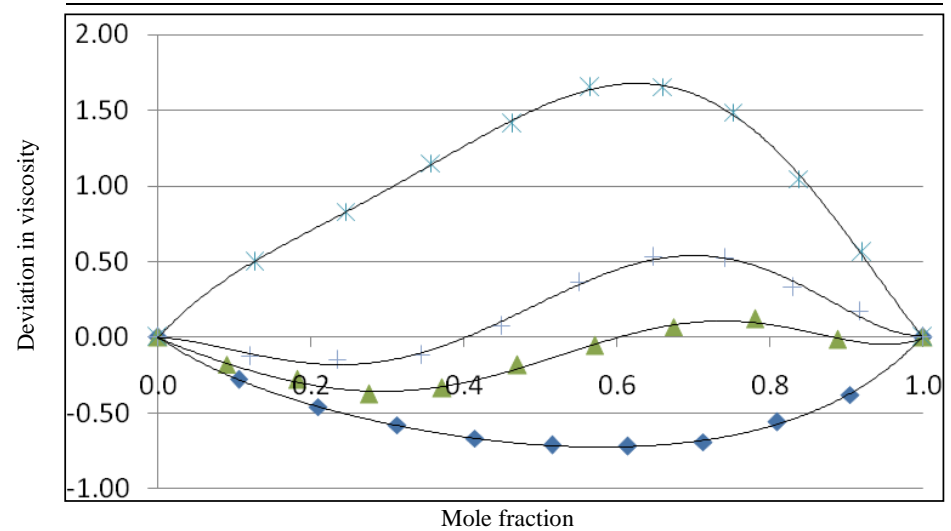

Figure 4. Variation of $\Delta \eta$ for $\left(\mathrm{x}_{1}\right)$ OCP + (1-x) PN (Ж), MIBK (X), EMK ( $\left.\Delta\right)$ and CYN ( $\left.\mathbf{a}\right)$ at $298.15 \mathrm{~K}$

Regression results, the coefficient Ai along with correlation coefficient $\mathrm{R}^{2}$, for the $\mathrm{V}^{\mathrm{E}}$ and $\Delta \eta$ of binary liquids along with correlation coefficient $\left(\mathrm{R}^{2}\right)$ are illustrated in Table 4 and 5.

$$
\text { The dependence of } \mathrm{V}^{\mathrm{E}} / \Delta \eta=\Sigma \text { Ai } \mathrm{x}_{1}{ }^{\mathrm{i}}(\mathrm{i}=0,1,2,3)
$$

Table 4. Regression results for the $\mathrm{V}^{\mathrm{E}}$ of binary liquids at $298.15 \mathrm{~K}$ along with correlation coefficients $\left(\mathrm{R}^{2}\right)$

\begin{tabular}{ccccccccc}
\hline System & $\mathrm{A}_{0}$ & $\mathrm{~A}_{1}$ & $\mathrm{~A}_{2}$ & $\mathrm{~A}_{3}$ & $\mathrm{~A}_{4}$ & $\mathrm{~A}_{5}$ & $\mathrm{~A}_{6}$ & $\mathrm{R}^{2}$ \\
\hline OCP+EMK & -0.008 & -4.973 & -49.20 & +195.0 & -287.5 & +208.9 & -62.26 & 0.998 \\
ODB+EMK & 0.028 & -30.48 & +136.2 & -423.5 & +732.7 & -621.2 & 206.2 & 0.996 \\
OCP+PN & -0.000 & -3.958 & +3.690 & +13.83 & -48.82 & +55.38 & -20.12 & 0.998 \\
ODB+PN & -0.001 & +0.662 & -86.52 & +287.2 & -364.2 & +200.4 & -37.56 & 0.999 \\
OCP+MIBK & +0.004 & +3.875 & -206.7 & +765.7 & -1207 & +917.7 & -273.3 & 0.998 \\
ODB+MIBK & -0.000 & -13.80 & -33.75 & +212.4 & -410.7 & +364.5 & -118.7 & 0.999 \\
OCP+CYN & +0.053 & -36.13 & +128.9 & -593.0 & +1188 & -979.5 & 291.4 & 0.996 \\
ODB+CYN & +0.012 & -7.112 & -142.6 & +585.5 & -1078 & +992.1 & -349.5 & 0.999 \\
\hline
\end{tabular}


Table 5. Regression results for the $\Delta \eta$ of binary liquids at $298.15 \mathrm{~K}$ along with correlation coefficients $\left(\mathrm{R}^{2}\right)$

\begin{tabular}{ccccccccc}
\hline System & $\mathrm{A}_{0}$ & $\mathrm{~A}_{1}$ & $\mathrm{~A}_{2}$ & $\mathrm{~A}_{3}$ & $\mathrm{~A}_{4}$ & $\mathrm{~A}_{5}$ & $\mathrm{~A}_{6}$ & $\mathrm{R}^{2}$ \\
\hline OCP+EMK & -0.001 & -2.123 & +3.066 & -7.870 & +44.73 & -69.53 & 31.73 & 0.991 \\
ODB+EMK & -0.000 & -0.399 & +1.956 & -7.438 & 14.72 & -13.36 & 4.523 & 0.999 \\
OCP+PN & -0.000 & +5.553 & -17.37 & +45.09 & -42.66 & -1.863 & 11.26 & 0.999 \\
ODB+PN & -0.005 & +0.108 & -0.117 & +1.854 & -5.479 & +5.742 & -2.107 & 0.999 \\
OCP+MIBK & -0.003 & -0.242 & -9.324 & +36.99 & -24.30 & -23.93 & 20.81 & 0.991 \\
ODB+MIBK & +0.000 & -0.123 & +0.292 & -0.571 & +0.784 & -0.063 & -0.318 & 0.993 \\
OCP+CYN & +0.000 & -3.411 & +9.077 & -21.10 & +33.10 & -27.30 & 9.641 & 0.999 \\
ODB+CYN & -0.000 & -0.102 & -0.325 & +1.481 & -2.114 & +1.541 & -0.480 & 0.997 \\
\hline
\end{tabular}

\section{Conclusion}

The computed data provides evidence for the existence of interactions between OCP and ODB with studies ketones.

\section{References}

1. Ezhil Pavai R, Vasantharani P and Kannappan A N, Indian J Pure Appl Phys., 2007, 45, 587.

2. $\quad$ Ali A, Yasmin A and Nain A K, Indian J Pure Appl Phys., 2002, 40, 315-322.

3. The Merck Index, An Encyclopedia of Chemicals, Drugs and Biologicals, $14^{\text {th }}$ Ed., 2006.

4. Riddick J A, Bunger W B and Sakano T K, Organic Solvents. Physical Properties and Methods of Purification, $4^{\text {th }}$ Ed., Wiley-Interscience, New York, 1986.

5. $\quad$ Fermeglia M and Lapasin J, J Chem Eng Data, 1988, 33, 415.

6. Dernini S, Polcaro A M, Ricci P F and Marongiu B J, J Chem Eng Data, 1987, 32, 194.

7. Cheng N L and Hu S W, Solvents Handbook, $1^{\text {st }}$ Ed., Chemical Engineering Press, Beijing, 1986.

8. Radojkovic, N, Tasic A, Grozdanic D, Djordjevic B and Malic D, J Chem Thermodyn, 1977, 9, 349-352.

9. $\quad$ Grunberg L, Trans Faraday Soc., 1954, 50, 1293.

10. Nikam P S and Shewale R P, Sawant A B and Hasan M, J Chem Eng Data., 2005, 50, 487.

11. Ravichandran G, Rao A S and Nambinarayanan J K, Indian J Pure Appl Phys., 1994, 32, 59.

12. Searles S and Tamres M, J Am Chem Soc., 1951, 73, 3704.

13. Shelar R N, Savale V S, Borse R Y, Hasan Mehdi and Sawant A B, Indian J Pure Appl Phys., 2008, 46, 552.

14. Chaudhary A, Gurung B B and Roy M N, J Indian Chem Soc., 2004, 81, 330. 